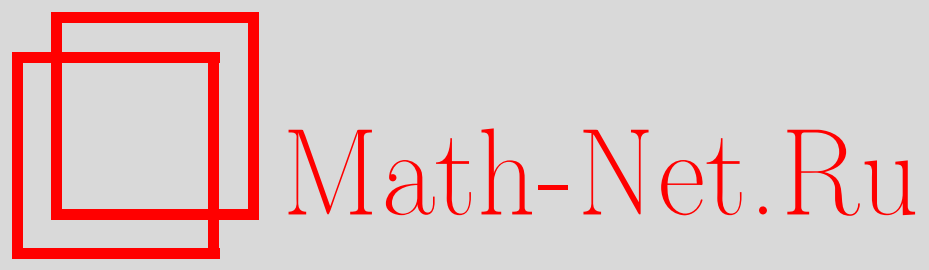

В. И. Драгович, Решения уравнения Янга и алгебраические кривые рода, большего 1, Функи. анализ и его прил., 1997, том 31, выпуск 2, 70-73

DOI: https://doi.org/10.4213/faa464

Использование Общероссийского математического портала MathNet.Ru подразумевает, что вы прочитали и согласны с пользовательским соглашением

http://www . mathnet.ru/rus/agreement

Параметры загрузки:

IP: 54.81 .137 .203

26 апреля 2023 г., 13:51:41

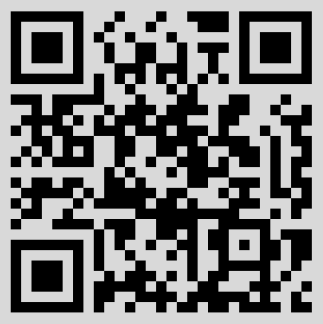




\title{
ЛитеРАтУРА
}

1. Арнольд В. И. Дополнительные главы теории обыкновенных дифференциальных уравнений. Наука, М., 1978. 2. Аносов Д. В. Геодезические потоки на замкнутых римановых многообразиях отрицательной кривизны. Труды МИАН им. В. А. Стеклова, 90, 1967. 3. Arrowsmith D. K., Cartwright J. H. E., Lansbury A. N., Place C. M. Internat. J. Bifurcation and Chaos, 3, No. 4, 803-842 (1993). 4. Богданов Р. И. Локальная орбитальная эквивалентность векторных полей на плоскости. Изд-во МГУ, 1993. 5. Ильяшенко Ю. С., Яковенко С. Ю.УМН, 46, №1, 3-40 (1991).

Московский государственный университет им. М. В. Ломоносова, Поступило в редакцию Институт ядерной физики

7 июня 1995 г.

УДК $517.9+513.015 .7$

\section{Решения уравнения Янга и алгебраические кривые рода, большего 1}

\author{
(c) 1997. В. И. ДРАГОвИч
}

В конце восьмидесятых годов Бакстер и др. (см. [1]), исследуя так называемую модель Поттса, нашли решения уравнения Янга

$$
R L L^{\prime}=L^{\prime} L R
$$

и уравнения Янга-Бакстера

$$
R^{12}\left(\Theta_{1}\right) R^{13}\left(\Theta_{2}\right) R^{23}\left(\Theta_{3}\right)=R^{23}\left(\Theta_{3}\right) R^{13}\left(\Theta_{2}\right) R^{12}\left(\Theta_{1}\right)
$$

со спектральным параметром, который параметризован кривой рода, большего 1. Доказано, что такие решения не удовлетворяют условию $\Theta_{3}=\Theta_{2}-\Theta_{1}$, и поэтому казалось, что они никак не связаны с уже известными решениями.

Но В. В. Бажанов и Ю. Г. Строганов нашли следуюшую связь (см. [2]). Они решали уравнение $(1)$, где в качестве $(4 \times 4)$-матрицы $R$ была взята $R$-матрица шестивершинной модели $R=R_{X X Z}$, и искали неизвестные $(2 n \times 2 n)$-матрицы $L$ и $L^{\prime}$. (Дополнительно был задан вид матриц $L$ и $L^{\prime}: L(x)=x L_{+}+x^{-1} L_{-}$.) Затем они рассматривали уравнение (1) с полученными таким образом матрицами $L$ и $L^{\prime}$ как уравнение с неизвестной $\left(n^{2} \times n^{2}\right)$-матрицей $R$. Выяснилось, что в качестве решения появляется именно $R$-матрица модели Поттса.

В этой работе мы исследуем общие решения уравнения (1), где $R$ - матрица размера $n^{2} \times n^{2}$, а $L$ и $L^{\prime}$ - матрицы размера $2 n \times 2 n$. Полученные матрицы будут иметь спектральные кривые рода, большего 1 , с нетривиальной группой автоморфизмов. Напомним, что в случае модели Поттса появляется кривая Ферма, которая, как известно, имеет нетривиальную группу автоморфизмов.

1. Спектральные кривые. Алгебро-геометрическая параметризация четномерных матриц, основанная на понятии вакуумных векторов и связанных с 
ними спектральных кривых, была предложена Кричевером (см. [3]). Любую четномерную матрицу $L$ размера $2 n \times 2 n$ можно рассматривать как $(2 \times 2)$-матрицу, элементы которой являются $(n \times n)$-матрицами. Другими словами, $L$ рассматривается как отображение $\mathbb{C}^{n} \otimes \mathbb{C}^{2} \rightarrow \mathbb{C}^{n} \otimes \mathbb{C}^{2}$. Греческими символами будем обозначать блок, а латинскими - место в блоке. Назовем вакуумньми векторами элементы пространства $\mathbb{C}^{n} \otimes \mathbb{C}^{2}$ вида $X \otimes U$, которые $L$ отображает в элементы того же вида $Y \otimes V$, т.е. $L(X \otimes U)=Y \otimes V$.

Вакуумные векторы параметризуются кривой $\Gamma$, которая задана уравнением

$$
P(u, v)=\operatorname{det}\left(L_{j}^{i}(u, v)\right)=0
$$

где $L_{j}^{i}=\tilde{V}^{\beta} L_{j \beta}^{i \alpha} U_{\alpha}, \tilde{V}=(1,-v), X_{n}=U_{2}=Y_{n}=V_{2}=1$ и $U_{1}=u, V_{1}=v$. Кривая $\Gamma$ называется спектральной кривой матрицы $L$.

Лемма 1. В общем случае род кривой Г будет равен $(n-1)^{2}$. Векторфункиии $X$ и имеют дивизорь степени $n^{2}-n$, а $U$ и $V$ имеют дивизорь степени $n$, причем

$$
D_{X}+D_{U} \sim D_{Y}+D_{V}
$$

Верно и обратное.

Лемма 2. Пусть Г-кривая рода $(n-1)^{2}$, пусть $X, Y$ и $U, V-$ заданные на ней вектор-функиии с дивизорами степеней $n^{2}-n$ u $n$ coответственно, и пусть $D_{X}+D_{U} \sim D_{Y}+D_{V}$. Тогда существует однозначно, $c$ точностью до умножения на константу, определенная матрица L размера $2 n \times 2 n$, такая, что

$$
L(X \otimes U)=h(Y \otimes V)
$$

әде $h$ - функция с дивизором нулей $D_{Y}+D_{V}$ и дивизором полюсов $D_{X}+D_{U}$, возникаюшая из условия нормировки.

2. Уравнение Янга с $(2 n \times 2 n)-,(2 n \times 2 n)$ - и $\left(n^{2} \times n^{2}\right)$-матрицами. Рассмотрим уравнение Янга

$$
L_{p \beta}^{\prime k \gamma} L_{q \gamma}^{l \alpha} R_{k l}^{i j}=R_{p q}^{k l} L_{k \beta}^{i \gamma} L_{i \gamma}^{\prime j \alpha}
$$

где латинские индексы принадлежат множеству $\{1, \ldots, n\}$, а греческие - множеству $\{1,2\}$. Пусть $P(u, v)=0$ и $P^{1}(u, v)=0-$ уравнения кривых $\Gamma$ и $\Gamma^{1}$, которые являются спектральными для матриц $L$ и $L^{1}$. Абсолютно так же, как это было показано Кричевером в случае $(4 \times 4)$-матриц, можно доказать, что для любого $n$ из (3) следует, что соответствия $P(u, v)=0, P^{1}\left(u^{1}, v^{1}\right)=0$ коммутируют в смысле композиции. Действительно, имеем

$$
\begin{aligned}
& L_{p \beta}^{\prime k \gamma} L_{q \gamma}^{l \alpha} R_{k l}^{i j}\left(R^{-1}\left(X^{1}(v, w) \otimes X(u, v)\right) \otimes U\right) \\
& \quad=h(u, v) h_{1}(v, w)\left(Y^{1}(v, w) \otimes Y(u, v) \otimes W\right), \\
& \left.R_{p q}^{k l} L_{k \beta}^{i \gamma} L_{l \gamma}^{\prime j \alpha}\left(X(\tilde{v}, w) \otimes X^{1}(u, \tilde{v}) \otimes U\right)\right) \\
& \quad=h_{1}(u, \tilde{v}) h(\tilde{v}, w) R\left(Y(\tilde{v}, w) \otimes Y^{1}(u, \tilde{v})\right) \otimes W
\end{aligned}
$$


В первом уравнении $u, v, w$ удовлетворяли равенствам $P(u, v)=0$, $P^{1}(v, w)=0$, а во втором $u, \tilde{v}, w$ удовлетворяли $P^{1}(u, \tilde{v})=0, P(\tilde{v}, w)=0$.

Так мы получаем следующее утверждение.

ПРЕДЛОЖЕНИЕ 1. Равенства $P(u, v)=0 u P^{1}\left(u^{1}, v^{1}\right)=0$ задают коммутирующие в смысле композиции соответствия.

ПРЕДЛОЖЕНИЕ 2. Матрица $R$ размера $n^{2} \times n^{2}$ имеет вакуумнье векmopbl.

Заметим, что в случае $n>2$ в общем положении существование вакуумных векторов невозможно. В этом можно убедиться при рассмотрении вложения Сегре.

ПРЕДЛОЖЕНИЕ 3. Пусть задана кривая $\Gamma$ рода $(n-1)^{2}$ и четьре $n$-компонентные вектор-функиии $X, U, Y, V c$ дивизорами степени $n^{2}-n$, для которьх $D_{X}+D_{U} \sim D_{Y}+D_{V}$. Тогда, с точностью до умножения на константу, однозначно определена матрица $R$ размера $n^{2} \times n^{2}$, такая, что имеет место равенство

$$
R(X \otimes U)=h(Y \otimes V) .
$$

ДоказАтельство. Пусть $D=D_{X}+D_{U}$. Из теоремы Римана-Роха имеем

$$
\operatorname{dim} L(D)=2\left(n^{2}-n\right)-(n-1)^{2}+1=n^{2} .
$$

Таким образом, координатные функции произведений $X \otimes U$ и $Y \otimes V$ составляют два базиса пространства $L(D)$, и мы определяем единственную матрицу перехода от одного базиса к другому, а это как раз матрица $L$, которую мы искали.

Тот факт, что равенства $P(u, v)=0$ и $P^{1}\left(u^{1}, v^{1}\right)=0$ коммутируют, дает две параметризации спектральной кривой $\Gamma$ матрицы $L^{\prime}: P^{1}(u, \tilde{v})=0$ и $P^{1}(v, w)=0$, которые определяют морфизм кривой $\Gamma:(u, \tilde{v}) \mapsto(v, w)$. Таким образом, мы получаем следующее утверждение:

ТЕоремА 1. Спектральная кривая Г матриць L, которая удовлетворяет уравнению Янаа, имеет нетривиальную группу автоморфизмов.

Нас интересует случай $n>2$, т.е. $g(\Gamma) \geqslant 4$. Известно (см. [4]), что в случае кривых рода $g$, большего 4 , сушествует только конечное число таких кривых с нетривиальной группой автоморфизмов, род которых ограничен числом 84 $(g-1)$. Результат теоремы 1, конечно, согласуется с моделью Поттса. Кривая, которая в этом случае возникает, является кривой Ферма и имеет нетривиальную группу автоморфизмов.

Из равенств (4) и (5) имеем следующие представления вакуумных векторов $R$-матрицы решения уравнения Янга (3) с данными матрицами $L$ и $L^{\prime}$ размера $2 n \times 2 n$.

TЕОРема 2. Пусть $X u X^{1}, Y u Y^{1}$ суть n-компонентные векторфункиии на кривой $\Gamma$ рода $(n-1)^{2}$, которье участвуют в представлении вакуумных векторов матрии $L$ и $L^{\prime}$ размера $2 n \times 2 n$. Тогда если матрииа $R$ размера $n^{2} \times n^{2}$ является решением уравнения (3), то она имеет следуюшие вакуумные представления:

$$
R X \cdot g_{1} \otimes X^{1}=X^{1} \cdot g_{2} \otimes X, \quad R Y \cdot g_{1} \otimes Y^{1}=Y^{1} \cdot g_{2} \otimes Y,
$$

zдe $g_{1}, g_{2} \in$ Aut $\Gamma$. 
Построение решений в описанной алгебро-геометрической ситуации является предметом дальнейших исследований.

Автор выражает благодарность Б. А. Дубровину и А. П. Веселову за полезные обсуждения.

\section{ЛИТЕРАТУРА}

1. Baxter J., Perk J. H. H., Yang H. A. Phys. Lett. A, 128, No. 3, 4 (1988). 2. Bazhanov V. V., Stroganov Yu. G. Chiral Potts Model as a Descendant of the SixVertex Model. Preprint, 1989. 3. Кричевер И. М. Функц. анализ и его прил., 15, 22-35 (1981). 4. Гриффитс П., Харрис И. Принципы алгебраической геометрии. Мир, М., 1978.

Математический институт,

Кнез Михайлова 35, 11000 Белград, Югославия

Поступило в редакцию 15 августа 1995 г.

УдК 514.853

\section{Особенности контакта окружностей с поверхностями и флаги}

(c) 1997. В. М. ЗАКАЛЮКИН

Дискриминанты групп, порожденных отражениями, появляются в качестве бифуркационных диаграмм в различных классификационных задачах теории особенностей. Еще одна достаточно естественная задача такого типа, по-видимому, не изученная ранее, связана с особенностями контакта окружностей в трехмерном евклидовом пространстве с заданной поверхностью общего положения [1]. Она находит приложения, например, в компьютерной графике [2] и вкладывается в более общую задачу классификации особенностей контакта подмногообразия в $\mathbb{R}^{n}$ с выбранным в этом пространстве полным или неполным флагом аффинных подпространств. Оказывается, что классы таких особенностей тесно связаны с краевыми особенностями, особенностями функций на многообразиях с углами [3], особенностями проектирований на прямую полных пересечений [4], уплощениями кривых [5] и т.д.

1. Пусть $S^{2}$ - некоторая сфера в евклидовом пространстве $\mathbb{R}^{3}$, и пусть $M_{S}^{3}$ - трехмерное многообразие всех окружностей (включая окружности нулевого радиуса), лежаших на сфере $S$. Заметим, что $M_{S}^{3}$ диффеоморфно пространству, полученному из шара $B$, ограниченного сферой $S, \sigma$-раздутием его центра. Действительно, отображение, сопоставляющее окружности ее центр, является диффеоморфизмом вне множества больших кругов, диффеоморфного $\mathbb{R} P^{2}$ и отображаемого в центр шара $B$.

Пусть Г- некоторая гладкая поверхность в $\mathbb{R}^{3}$. Бифуркационным множеством $D(\Gamma, S) \subset M_{S}$ назовем множество окружностей, не трансверсальных $\Gamma$. Особенности бифуркационных множеств $D(\Gamma, S)$ опишем с помощью следующей конструкции. На пространстве пар $(f, g): \mathbb{R}^{2} \rightarrow \mathbb{R}$ гладких функций, заданных

\footnotetext{
^ Работа выполнена при поддержке грантов РФФИ 96-01-00710 и INTAS 4373.
} 